\title{
Higher-Dimensional Box Integrals
}

\author{
Jonathan M. Borwein, O-Yeat Chan, and R. E. Crandall
}

\section{CONTENTS}

1. Background and Nomenclature

2. State of the Box-Integral Art

3. Complete Resolution of Box Residues

4. Some Key Integrals

5. Overcoming the Blockade at Five Dimensions

6. The Concept of Expression Entropy

7. Six-Dimensional Box Integrals

8. Theory of Hyperdegree

9. Conclusion

10. Appendix I: The functions $\boldsymbol{F}^{ \pm}$and $G^{ \pm}$

11. Appendix II: Explicit forms for $J(n), 0 \leq n \leq 4$, and $C_{3,0}(-2, A)$

Acknowledgments

References
2000 AMS Subject Classification: 33B30, 11Y60

Keywords: Box integral, trilogarithm, arctan integral
Herein, with the aid of substantial symbolic computation, we solve previously open problems in the theory of $n$-dimensional box integrals $B_{n}(s):=\left\langle|\vec{r}|^{s}\right\rangle, \vec{r} \in[0,1]^{n}$. In particular, we resolve an elusive integral called $\mathcal{K}_{5}$ that previously acted as a "blockade" against closed-form evaluation in $n=5$ dimensions. In consequence, we now know that $B_{n}$ (integer) can be given a closed form for $n=1,2,3,4,5$. We also find the general residue at the pole at $s=-n$, this leading to new relations and definite integrals; for example, we are able to give the first nontrivial closed forms for six-dimensional box integrals and to show hyperclosure of $B_{6}$ (even). The Clausen function and its generalizations play a central role in these higher-dimensional evaluations. Our results provide stringent test scenarios for symbolicalgebra simplification methods.

\section{BACKGROUND AND NOMENCLATURE}

Recent papers by Bailey, Borwein, and Crandall [Bailey et al. 07, Bailey et al. 09]-stemming from the historical work of [Anderson et al. 76] - have exhibited new results on the computation and analysis of box integrals, these being expectations of radius and separation, respectively, within an $n$-cube. We write formally $B_{n}(s):=\left\langle|\vec{r}|^{s}\right\rangle$, $\Delta_{n}(s):=\left\langle|\vec{r}-\vec{q}|^{s}\right\rangle$, with specific definitions

$$
\begin{aligned}
B_{n}(s) & :=\int_{\vec{r} \in[0,1]^{n}}|\vec{r}|^{s} \mathcal{D} \vec{r} \\
& =\int_{0}^{1} \cdots \int_{0}^{1}\left(r_{1}^{2}+\cdots+r_{n}^{2}\right)^{s / 2} d r_{1} \cdots d r_{n} \\
\Delta_{n}(s) & :=\int_{\vec{r}, \vec{q} \in[0,1]^{n}}|\vec{r}-\vec{q}|^{s} \mathcal{D} \vec{r} \mathcal{D} \vec{q} \\
& =\int_{0}^{1} \cdots \int_{0}^{1}\left(\left(r_{1}-q_{1}\right)^{2}+\cdots+\left(r_{n}-q_{n}\right)^{2}\right)^{s / 2} \\
& d r_{1} \cdots d r_{n} d q_{1} \cdots d q_{n}
\end{aligned}
$$

Introduced in [Bailey et al. 09] is a useful function, a kind of generalized box integral:

$$
C_{m, 0}(s, a):=\int_{\vec{r} \in[0,1]^{m}}\left(a+r^{2}\right)^{s / 2} \mathcal{D} \vec{r} .
$$


Let us list some relevant known facts and interrelations among these functions. It is important to observe that $B_{n}(s), \Delta_{n}(s)$ have well-defined analytic continuations over the entire complex $s$-plane, with $B_{n}(s)$ having a solitary pole at $s=-n$ and $\Delta_{n}(s)$ having exactly $n+1$ poles, at $s=-2 n,-2 n+1, \ldots,-n$. In particular, though something like $B_{4}(-5)$ does not converge as a literal box integral, its value exists unambiguously, and is known in closed form, namely as the negative value $-\sqrt{8} \arctan (1 / \sqrt{8})$. References [Bailey et al. 07, Bailey et al. 09] establish the analytic properties together with the following results:

1. $B, C$ relations:

$$
\begin{aligned}
B_{n}(s) & =\frac{n}{n+s} C_{n-1,0}(s, 1), \\
B_{n}(-n-1) & =-n C_{n-2,0}(1-n, 2), \\
\operatorname{Res}_{n} & =n C_{n-1}(-n, 1),
\end{aligned}
$$

where $\operatorname{Res}_{n}:=\lim _{\epsilon \rightarrow 0} \epsilon B_{n}(-n+\epsilon)$ is the residue of $B_{n}$ at the solitary pole $(s=-n)$.

2. Absolutely convergent analytic series for $B$, with pole at $s=-n$ :

$$
B_{n}(s)=\frac{n^{1+s / 2}}{s+n} \sum_{k \geq 0} \gamma_{n-1, k}\left(\frac{2}{n}\right)^{k},
$$

where the $\gamma_{m, k}$ are certain fixed coefficients defined by the recurrence [Crandall 10]

$$
(1+2 k / m) \gamma_{m, k}=(k-1-s / 2) \gamma_{m, k-1}+\gamma_{m-1, k}
$$

for $m, k \geq 1$, this recurrence being ignited by $\gamma_{0, k}:=\delta_{0, k}$, $\gamma_{m, 0}:=1$.

3. Recurrence relations:

$$
\begin{aligned}
C_{0,0}(s, a):= & a^{s / 2}, \\
C_{n, 0}(s, 0)= & B_{n}(s), \\
a s C_{m, 0}(s-2, a)= & (s+m) C_{m, 0}(s, a) \\
& -m C_{m-1,0}(s, a+1), \\
(n+s)(n+s-1) B_{n}(s)= & s(n+s-2) B_{n}(s-2) \\
& +n(n-1) C_{n-2,0}(s, 2) .
\end{aligned}
$$

\section{STATE OF THE BOX-INTEGRAL ART}

We have enumerated some important relations; presently, we summarize known results in regard to specific dimensions.

To begin our summary, we note a recent definition of a class of closed forms. Following the treatment [Bailey et al. 09], we define a complex number to be hyperclosed if it belongs to the ring of hyperclosure. This ring is built as follows (see [Bailey et al. 09] and [Borwein and Crandall 10] for more details). Consider generalized hypergeometric evaluations

$$
x=\sum_{n \geq 0} c_{n} z^{n},
$$

where $z$ is algebraic, $c_{0}$ is rational, and $c_{n}=\frac{p(n)}{q(n)} c_{n-1}$, where $p$ and $q$ are polynomials with integer coefficients. Then the ring of hyperclosure is generated by all such evaluations $x$ under $(+, \cdot)$. We say that any element of the ring is hyperclosed. So for example, $\pi, \pi+\log (2+\sqrt{7})$, $\operatorname{Li}_{2}(1 / \sqrt{5})+(\log 2)(\log 3)$, are all hyperclosed. Incidentally, lest one think the ring of hyperclosure is just too broad, note that said ring is countable [Bailey et al. 09].

Known results on box integrals include the following:

1. Resolution of the $\Delta_{n}$ : It was shown in the treatment [Bailey et al. 09, Theorem 7] that if all $B_{m}(s)$ and all residues $\operatorname{Res}_{m}$ are known for $m \in[1, n]$, then $\Delta_{n}(s)$ is known. In that previous treatment, a form for the box residues is conjectured and some low-lying residues are given exactly. Happily, in the present work we shall establish a closed form for all $\operatorname{Res}_{m}$ with $m \in \mathbb{N}$. Thus, the problem of evaluating the $\Delta_{n}$ evaporates entirely, being replaced by the problem of evaluating the $B_{n}$.

2. Hyperclosure in dimensions 1,2,3,4: It is known that for any integer $k$, all $B_{1}(k), B_{2}(k), B_{3}(k), B_{4}(k)$ are hyperclosed (exemplary closed forms appear in [Bailey et al. 09]). The same knowledge exists for $\Delta_{n}(k), n=1,2,3,4$, by the arguments of the previous item.

3. Dimension 5: It has been shown in previous work that for integer $k \neq-2,-4$, the box integral $B_{5}(k)$ is hyperclosed. The two exceptional $k$ arguments have amounted to a "blockade" - with the obstacle amounting to a single, tough integral called $\mathcal{K}_{5}$, which integral we resolve below. This resolution of $\mathcal{K}_{5}$ establishes hyperclosure of all $B_{5}$ (integer), and perforce all $\Delta_{5}$ (integer).

4. Dimension 6: Previous to our present treatment, not a single nontrivial $B_{6}$ (integer) had been evaluated in closed form. (We say "nontrivial" here because, of course, $B_{n}(2 h)$ for a positive integer $h$ is trivially rational.) But we are able to exhibit later in this paper the closed form for $B_{6}(-4)$, thus breaking the 
dimension-6 impasse. Moreover, using $B_{6}(-4)$ as a recursive pivot, we now know that $B_{6}$ (even) are all hyperclosed. Virtually nothing is known, however, about $B_{6}$ (odd).

5. Very high dimension: An algorithm has been discovered [Crandall 10] that uses a series of the form (1-6) to resolve $D$ digits of a box integral $B_{n}(s)$ in $O\left(n^{2} D\right)$ operations, where the implied big- $O$ constant depends only on $s$. D. H. Bailey has employed said algorithm to achieve a dimension-one-million box value to one hundred good decimals, the value starting out

$$
\begin{array}{r}
B_{1000000}(1)=577.3502114545720399775340875203 \\
6227457448125926146101942964 \ldots .
\end{array}
$$

It is evident that the art of calculation of the $B_{n}$ is vastly ahead of the corresponding symbolic art.

\section{COMPLETE RESOLUTION OF BOX RESIDUES}

The general box-residue evaluation can be effected as follows. For $n$ dimensions, the residue will be

$$
\operatorname{Res}_{n}:=\lim _{\delta \rightarrow 0^{+}} \delta B_{n}(-n+\delta)=\lim _{\delta \rightarrow 0^{+}} \delta \int_{[0,1]^{n}} r^{-n+\delta} \mathcal{D} \vec{r} .
$$

But the volume element is

$$
\mathcal{D} \vec{r}=r^{n-1} d r \mathcal{D} \vec{\Omega}
$$

where $\mathcal{D} \vec{\Omega}$ is a suitable definition of solid angle for $n$ space. Thus the $r$ integral inside is

$$
\int_{0}^{R(\vec{\Omega})} \frac{1}{r^{1-\delta}} d r
$$

where $R(\vec{\Omega})$ is the extent of radius from the origin to the surface of the $n$-cube along the direction $\vec{\Omega}$. The integral is

$$
\frac{1}{\delta} R(\vec{\Omega})^{\delta}
$$

which when multiplied by the outside factor $\delta$ can be taken to be 1 . Thus, the residue comes down to being a piece (an $n$-orthant) of the surface area of the unit $n$ sphere, namely

$$
\operatorname{Res}_{n}=\frac{1}{2^{n-1}} \frac{\pi^{n / 2}}{\Gamma(n / 2)} .
$$

This knowledge of all box residues leads, as we shall see, to new closed forms.
Another application of the general residue value is as follows. The second author of this paper has conjectured that the pretty integral

$$
I_{n}:=\int_{[0, \pi / 4]^{n}} \frac{d \theta_{1} \cdots d \theta_{n}}{\left(1+\sec ^{2} \theta_{1}+\cdots+\sec ^{2} \theta_{n}\right)^{1 / 2}}
$$

is a rational multiple of $\pi^{n}$. This conjecture arose via numerical computation, then observation that the result matches sequence A002457 in Sloane's Online Encyclopedia [Sloane 09]. Here we prove the conjecture, giving as well the precise rational multiplier. First, it is a straightforward combinatorial result, via polar coordinates on each pair of integration variables in (1-3), that

$$
C_{2 n, 0}(-2 n-1,1)=\frac{2^{n}}{(2 n-1) ! !} \sum_{k=0}^{n}(-1)^{k}\left(\begin{array}{l}
n \\
k
\end{array}\right) I_{k} \frac{\pi^{n-k}}{4^{n-k}}
$$

But by relation (1-5), the left-hand side is just $\operatorname{Res}_{2 n+1} /(2 n+1)$, which is given by $(3-1)$. We now have

$$
\sum_{k=0}^{n}(-1)^{k}\left(\begin{array}{l}
n \\
k
\end{array}\right) \frac{I_{k}}{(\pi / 4)^{k}}=\frac{1}{2 n+1}
$$

The global solution to this recurrence system can be resolved by consequence of the binomial transform being an involution. We write

$$
\begin{aligned}
& \left\{\sum_{k=0}^{n}(-1)^{k}\left(\begin{array}{l}
n \\
k
\end{array}\right) \frac{I_{k}}{(\pi / 4)^{k}}=\frac{1}{2 n+1}, \quad \forall n \in N\right\} \\
& \quad \Leftrightarrow\left\{\left(\frac{\pi}{4}\right)^{n} \sum_{k=0}^{n}(-1)^{k}\left(\begin{array}{l}
n \\
k
\end{array}\right) \frac{1}{2 k+1}=I_{n}, \quad \forall n \in N\right\} .
\end{aligned}
$$

Summing the finite series on the right and simplifying, we obtain

$$
\int_{[0, \pi / 4]^{k}} \frac{d \theta_{1} \cdots d \theta_{k}}{\left(1+\sec ^{2} \theta_{1}+\cdots+\sec ^{2} \theta_{k}\right)^{1 / 2}}=\frac{k !^{2}}{(2 k+1) !} \pi^{k} .
$$

\section{SOME KEY INTEGRALS}

To overcome the aforementioned box-integral blockade at five dimensions and to move beyond into dimension- 6 cases, we now establish closed forms for some key definite integrals. It is evident from the $B, C$ relations in Section 1 that box integrals $B_{n}$ depend intimately on $C_{m, 0}$ integrals. We are thus interested in parameterized integrals $C_{m, 0}(s, A)$, where the parameter $A$ is a positive integer. ${ }^{1}$ The most important instance for our present purposes

\footnotetext{
${ }^{1}$ We choose to keep the second subscript 0 on $C_{m, 0}$ just for consistency with previous treatments such as [Bailey et al. 09].
} 
can be developed via straightforward polar integration as

$$
\begin{aligned}
C_{4,0}(-4, A)= & -J(A+2)+\frac{1}{16} \pi^{2} \log A-\frac{\pi}{2} G \quad(4-1) \\
+ & \frac{\pi}{4} \operatorname{Im}\left(\operatorname{Li}_{2}\left(\frac{i(A-2 \sqrt{A+1}+2)}{A}\right)\right. \\
& \left.+\operatorname{Li}_{2}\left(\frac{i(A+2 \sqrt{A+1}+2)}{A}\right)\right) ;
\end{aligned}
$$

see also (11-7). Here $J$ is in turn a highly difficult integral that we define by

$$
J(t):=\int_{[0,1]^{2}} \frac{\log \left(t+x^{2}+y^{2}\right)}{\left(1+x^{2}\right)\left(1+y^{2}\right)} d x d y .
$$

We are interested in whether $J(t)$ is hyperclosed for algebraic $t$; accordingly, we begin with a new, fundamental, result.

Lemma 4.1. For any complex parameter $t$ with $\operatorname{Re}(t) \geq 0$, we have

$$
\begin{aligned}
J(t)=-\frac{\log 2}{2} \operatorname{Re}( & \operatorname{Li}_{2}\left(\frac{2}{\sqrt{t-1}+1}\right) \\
& \left.+\operatorname{Li}_{2}\left(-\frac{2}{\sqrt{t-1}-1}\right)\right)+R(t)
\end{aligned}
$$

where

$$
\begin{aligned}
R(t):= & \int_{0}^{1} \log \left(\frac{1+x}{1-x}\right) \\
\times & \left(\frac{x \log t-x \log \left(t+x^{2}+1\right)}{t-1-x^{2}}\right. \\
& +(t-1) \frac{x \log \left(t+x^{2}+1\right)-x \log t-x \log \left(x^{2}+1\right)}{1-(t-1) x^{2}} \\
& \left.+\frac{x \log \left(t+x^{2}+1\right)}{x^{2}+1}-\frac{x \log \left(x^{2}+1\right)}{x^{2}}\right) d x,
\end{aligned}
$$

with $J$ interpreted at either of the special points $t=1,2$ via the limit of expression (4-2) for $t:=q+\frac{1}{1-i \epsilon}, q=0,1$, and $\epsilon \rightarrow 0^{+}$.

Proof. We evaluate the integral $J(t)$ via a series of changes of variables. We begin by making a change into polar coordinates $(r, \theta)$ and then applying partial fractions, and finally making the change of variables $x^{2}=\sec ^{2} \theta-1$ and $y=r^{2}$ to obtain

$$
\begin{aligned}
J(t) & =2 \int_{0}^{\pi / 4} d \theta \int_{0}^{\sec \theta} r d r \frac{\log \left(t+r^{2}\right)}{\left(1+r^{2} \cos ^{2} \theta\right)\left(1+r^{2} \sin ^{2} \theta\right)} \\
& =\lim _{\delta \rightarrow 1} \int_{0}^{\delta} \frac{d x}{1-x^{2}} \\
& \times \int_{0}^{x^{2}+1} \log (t+y) d y\left(\frac{1}{x^{2}+1+y}-\frac{1}{x^{-2}+1+y}\right) .
\end{aligned}
$$

The rest of the analysis (we omit many details here to conserve space) employs the relations

$$
\frac{d}{d x} \operatorname{Li}_{2}(-x)=-\frac{\log (1+x)}{x}
$$

and

$$
\frac{d}{d x} \operatorname{Li}_{2}(a / x)=\frac{\log (1-a / x)}{x},
$$

and the dilogarithm inversion relation

$$
\operatorname{Li}_{2}(-x)+\operatorname{Li}_{2}(-1 / x)=-\frac{\pi^{2}}{6}-\frac{\log ^{2} x}{2},
$$

along with rather intricate integration-by-parts and partial-fraction manipulations, all of this to obtain, finally, (4-2).

\section{OVERCOMING THE BLOCKADE AT FIVE DIMENSIONS}

The integral known as $\mathcal{K}_{5}$, which appears in the relation

$$
\begin{aligned}
B_{5}(-4)= & -5 \mathcal{K}_{5}-\frac{5}{2} \pi G+\frac{5}{4} \pi^{2} \log (1+\sqrt{2}) \\
& +\frac{5}{2} \pi \operatorname{Ti}_{2}(3-2 \sqrt{2})
\end{aligned}
$$

was not resolved in [Bailey et al. 09]. Here $\mathrm{Ti}_{2}$ is the generalized arctangent of order two [Lewin 81]. The literature definition can be cast as

$$
\begin{aligned}
\mathcal{K}_{5} & :=\int_{0}^{\pi / 4} \int_{0}^{\pi / 4} \log \left(1+\sec ^{2} a+\sec ^{2} b\right) d a d b \\
& =\int_{[0,1]} \frac{\log \left(3+x^{2}+y^{2}\right)}{\left(1+x^{2}\right)\left(1+y^{2}\right)} d x d y=J(3) .
\end{aligned}
$$

Thus if $J(3)$ is hyperclosed, then so is $B_{5}(-4)$. One can see clearly now why we have focused on the $J$ integral.

With Lemma 4.1 we have reduced the problem of evaluating $J(t)$, and perforce $\mathcal{K}_{5}=J(3)$, to that of evaluating $R(t)$. It will turn out that a necessary ingredient in our quest to pass the five-dimensional blockade will be the invocation of trilogarithms, which entities had not yet appeared in any previous closed forms for box integrals. 
To this end, we invoke parameterized definite integrals, namely the pair

$$
F^{ \pm}(c):=\int_{0}^{1} \frac{\log (1 \pm x)}{x+c} d x
$$

and the pair

$$
G^{ \pm}(b, c):=\int_{0}^{1} \frac{\log (1 \pm x) \log (x+b)}{x+c} d x .
$$

The closed forms for these four integrals are rather stupefying; we have relegated the closed-form displays to our Appendix I (Section 10), the salient point being that we shall find that the remaining integral $R(t)$ can be cast as a superposition of $F$ and $G$ forms.

Theorem 5.1. $J(t)$ is hyperclosed for algebraic $t$ with $\operatorname{Re}(t) \geq 0$. (An explicit closed form is indicated in the proof following, although as before, the special points $t=0,1$ need be handled as limiting values; see Appendix II (Section 11).)

Proof. We write $R(t)$ as four definite integrals

$$
R(t)=Y_{1}+Y_{2}+Y_{3}+Y_{4},
$$

with the $Y_{n}$ ordered as in the display of Lemma 4.1. To clarify, we have, for example,

$$
Y_{3}:=\int_{0}^{1} \log \left(\frac{1+x}{1-x}\right) \frac{x \log \left(t+x^{2}+1\right)}{x^{2}+1} d x .
$$

Taking this exemplary case, we have-upon logexpansion and partial fractions - the following:

$$
\begin{aligned}
Y_{3}=\int_{0}^{1} & (\log (1+x)-\log (1-x)) \\
& \times(\log (x+i \sqrt{t+1})+\log (x-i \sqrt{t+1})) \\
& \times \frac{1}{2}\left(\frac{1}{x+i}+\frac{1}{x-i}\right) d x .
\end{aligned}
$$

But this means that $Y_{3}$ is a superposition of $G^{+}, G^{-}$evaluations, as is the case for each of the $Y_{n}$ below if we also include $F$ functions as needed (which to avoid many line breaks are displayed in Figure 1), with each sum for an $F$-function performed over sign choices \pm , and for a $G$ function over all four possible sign choices \pm \pm . (One might object that $Y_{2}$ in particular involves integration over a branch singularity when $t>2$, but in fact the integrand for $Y_{2}$ can be seen to be finite and differentiable; that is, any branch-cut effects are canceled in the given superposition for $Y_{2}$.)
We therefore have a closed form based on the above closed forms for the $Y_{n}$, namely

$$
\begin{aligned}
J(t)= & -\frac{\log 2}{2} \operatorname{Re}\left(\operatorname{Li}_{2}\left(\frac{2}{\sqrt{t-1}+1}\right)\right. \\
& \left.+\operatorname{Li}_{2}\left(-\frac{2}{\sqrt{t-1}-1}\right)\right) \\
& +\left(R(t)=Y_{1}+Y_{2}+Y_{3}+Y_{4}\right) .
\end{aligned}
$$

For algebraic $t$ this is a hyperclosed representation of $J(t)$, since our appendix forms for $F^{ \pm}, G^{ \pm}$are hyperclosed for such $t$.

Because $\mathcal{K}_{5}=J(3)$ has been the sole obstacle to 5 -dimensional hyperclosure, we now have the following corollary.

Corollary 5.2. The integral $\mathcal{K}_{5}$ is hyperclosed, and therefore all box integrals $B_{5}$ (integer), and perforce $\Delta_{5}$ (integer), are hyperclosed.

The $J$ that are integral at integer arguments other than $t=3$ are interesting in their own right. Our Appendix II discusses closed-form developments for these cases.

\subsection{A Generalization of $J$}

We conclude this portion of the paper by placing $J(A)$ in a more general context. Recall that

$$
\begin{aligned}
B_{2 n+1} & (-s) \\
:= & \int_{[0,1]^{2 n+1}}\left(x_{1}^{2}+\cdots+x_{2 n+1}^{2}\right)^{-s / 2} d x_{1} \cdots d x_{2 n+1} \\
= & \frac{2 n+1}{2 n+1+s} C_{2 n, 0}(-s, 1) \\
= & \frac{2 n+1}{2 n+1+s} \\
& \times \int_{[0,1]^{2 n}}\left(1+x_{1}^{2}+\cdots+x_{2 n}^{2}\right)^{-s / 2} d x_{1} \cdots d x_{2 n} .
\end{aligned}
$$

For general $C_{2 n, 0}$, we may change pairs of variables into polar coordinates to obtain

$$
\begin{aligned}
C_{2 n, 0} & (-s, A) \\
:= & \int_{[0,1]^{2 n}}\left(A+x_{1}^{2}+x_{2}^{2}+\cdots+x_{2 n}^{2}\right)^{-s / 2} d x_{1} \cdots d x_{2 n} \\
= & \frac{2^{n}}{(2-s)(4-s) \cdots(2 n-s)} \sum_{k=0}^{n}(-1)^{n-k}\left(\begin{array}{l}
n \\
k
\end{array}\right) \\
& \times I_{n, k}(s, A) \frac{\pi^{n-k}}{4^{n-k}},
\end{aligned}
$$




$$
\begin{aligned}
Y_{1}= & \frac{1}{2}\left(\sum G^{+}( \pm i \sqrt{t+1}, \pm \sqrt{t-1})-\sum G^{-}( \pm i \sqrt{t+1}, \pm \sqrt{t-1})\right) \\
& -\frac{\log t}{2}\left(\sum F^{+}( \pm \sqrt{t-1})-\sum F^{-}( \pm \sqrt{t-1})\right) \\
Y_{2}= & -\frac{1}{2}\left(\sum G^{+}( \pm i \sqrt{t+1}, \pm 1 / \sqrt{t-1})-\sum G^{-}( \pm i \sqrt{t+1}, \pm 1 / \sqrt{t-1})\right) \\
& +\frac{1}{2}\left(\sum G^{+}( \pm i, \pm 1 / \sqrt{t-1})-\sum G^{-}( \pm i, \pm 1 / \sqrt{t-1})\right) \\
& +\frac{\log t}{2}\left(\sum F^{+}( \pm 1 / \sqrt{t-1})-\sum F^{-}( \pm 1 / \sqrt{t-1})\right) \\
Y_{3}= & \frac{1}{2}\left(\sum G^{+}( \pm i \sqrt{t+1}, \pm i)-\sum G^{-}( \pm i \sqrt{t+1}, \pm i)\right) \\
Y_{4}= & -G \pi+\frac{7}{4} \zeta(3)
\end{aligned}
$$

FIGURE 1. Resolution of key integrals in terms of $F^{ \pm}, G^{ \pm}$forms (see Appendix).

where

$$
I_{n, k}(s, A):=\int_{[0, \pi / 4]^{k}} \frac{d \theta_{1} \cdots d \theta_{k}}{\left(A+\sec ^{2} \theta_{1}+\cdots+\sec ^{2} \theta_{k}\right)^{s / 2-n}}
$$

provided that $s \neq 2,4, \ldots, 2 n$. The further change of variable $x_{i}^{2}=\sec ^{2} \theta_{i}-1$ brings us back to the unit $k$ cube, so that

$$
\begin{aligned}
& I_{n, k}(s, A) \\
& \quad=\int_{[0,1]^{k}} \frac{d x_{1}}{x_{1}^{2}+1} \cdots \frac{d x_{k}}{x_{k}^{2}+1} \frac{1}{\left(A+k+x_{1}^{2}+\cdots+x_{k}^{2}\right)^{s / 2-n}} .
\end{aligned}
$$

In the case $s=2 n$, we find that

$$
\begin{aligned}
B_{2 n+1}(-2 n)= & \frac{(-1)^{n-1}}{(2 n-1)(n-1) !} \\
& \times \sum_{k=0}^{n}(-1)^{n-k}\left(\begin{array}{l}
n \\
k
\end{array}\right) \frac{\pi^{n-k}}{4^{n-k}} J_{k}(k+1)
\end{aligned}
$$

where $J_{0}(A):=1$ and for $k \geq 1$ we have

$$
\begin{gathered}
J_{k}(A):=\int_{[0,1]^{k}} \frac{d x_{1}}{x_{1}^{2}+1} \cdots \frac{d x_{k}}{x_{k}^{2}+1} \log \left(A+x_{1}^{2}+\cdots+x_{k}^{2}\right) \\
=\int_{[0, \pi / 4]^{k}} \log \left(A+\tan ^{2} \theta_{1}+\cdots\right. \\
\left.+\tan ^{2} \theta_{k}\right) d \theta_{1} \cdots d \theta_{k}
\end{gathered}
$$

Moreover, $J_{k+1}(A)=\int_{0}^{\pi / 4} J_{k}\left(A+\tan ^{2} u\right) d u$.

To illustrate,

$$
J_{2}(A)=J(A)=\int_{0}^{1} \int_{0}^{1} \frac{\log \left(A+x^{2}+y^{2}\right)}{\left(1+x^{2}\right)\left(1+y^{2}\right)} d x d y
$$

and $J_{2}(3)=J(3)=\mathcal{K}_{5}$, while a less-difficult version of Lemma 4.1 shows that

$$
\begin{aligned}
J_{1}(A)= & \int_{0}^{\pi / 4} \log \left(A+\tan ^{2} t\right) d t \\
= & \frac{\pi}{4} \log (A-1)+\left(\theta-\frac{\pi}{4}\right) \log \left(\frac{\sqrt{A}-1}{\sqrt{A}+1}\right) \\
& +\frac{1}{2} \mathrm{Cl}_{2}(\theta)-\frac{1}{2} \mathrm{Cl}_{2}(\theta-\pi)-G,
\end{aligned}
$$

where $\theta:=\arctan \left(\frac{A-1}{2 \sqrt{A}}\right)=2 \arctan \left(\frac{\sqrt{A}-1}{\sqrt{A}+1}\right)$.

Hence $(5-3)$ and $(5-4)$ show that $B_{7}(-6)$ is hyperclosed iff $J_{3}(4)$ is, which suggests that the evaluation of $B_{2 n+1}(-2 n)$ can be achieved in terms of $\mathrm{Cl}_{k}$ for $2 \leq k \leq n+1$.

\section{THE CONCEPT OF EXPRESSION ENTROPY}

Though box integrals $B_{5}$ (integer) are now known to be hyperclosed, it is surprising that the closed form for something like $B_{5}(-6)$ occupies one line of typical typesetting, yet our initial closed form for $J(3)$ (essentially $B_{5}(-4)$ ) from Theorem 5.1 had on the order of $10^{5}$ characters. Furthermore, different symbolic languages would use differing character counts to express $J(3) .^{2}$

We are motivated thus to introduce the notion of expression entropy. This will be the number of binary bits inherent in an expression. And we found a very simple practical means of measuring such entropy: run an expression as a text file through an established entropy compressor.

For example, our initial $J(3)$ expression, when entropy-compressed, reduced down to about $5 \cdot 10^{4}$ bits.

\footnotetext{
${ }^{2}$ In our case, Maple and Mathematica were used extensively throughout our efforts at expression reduction.
} 
Note that an initial $10^{5}$ text characters is on the order of $10^{6}$ bits, so this is significant reduction. One way to think of how expression entropy works is to consider that a good entropy compressor will essentially not care whether dilogarithms are written $\operatorname{Li} 2(\ldots)$ or PolyLog $[2, \ldots]$, since the compressor is typically looking for common strings.

The reason why the expression-entropy concept is useful can be inferred from our appendix commentary - note that $J(1), J(2), J(3)$, and $J(4)$ each start out as many pages of text, and even at current reductions, $J(4)$ still barely fits on a single page. Yet $J(2)$ has reduced to a single line. The current work makes it abundantly clear that much work remains to be done regarding effective closed forms. Both Maple and Mathematica were able to rapidly confirm symbolic closed forms numerically but were unable in every case to produce symbolically humanly convenient expressions.

\section{SIX-DIMENSIONAL BOX INTEGRALS}

\section{$7.1 \quad B_{6}$ (even)}

For general dimension $n$, the recurrence (1-9) with $s=$ $-n+2$ yields

$2 B_{n}(-n+2)=(-n+2) \operatorname{Res}_{n}+n(n-1) C_{n-2,0}(2-n, 2)$.

Importantly, this reduces this box-integral $B_{n}$ to an integral of dimension $(n-2)$. For example, the previously unresolved $B_{6}(-4)$ can now be written using the known residue $\operatorname{Res}_{6}$ as

$$
B_{6}(-4)=-\frac{\pi^{3}}{32}+15 C_{4,0}(-4,2) .
$$

From knowledge of (4-1) and Theorem 5.1, it follows that $B_{6}(-4)$ is hyperclosed.

But we can carry this 6-dimensional effort yet further. The recurrence relations such as (1-8), (1-9) give us the two key recurrences

$$
2 s C_{4,0}(s-2,2)=(s+4) C_{4,0}(s, 2)-4 C_{3,0}(s, 3)
$$

and

$$
(s+6)(s+5) B_{6}(s)=s(s+4) B_{6}(s-2)+30 C_{4,0}(s, 2) .
$$

These recurrences contain enough magic to resolve all of the box integrals $B_{6}$ (even). Indeed, pivoting on our knowledge of $B_{6}(-4)$ as given above, (7-2) leads us to

$$
B_{6}(-2)=-\frac{2}{3} B_{6}(-4)-\frac{\pi^{3}}{96}+5 C_{3,0}(-2,3) .
$$

Happily, it turns out that the techniques used in our main Lemma 4.1 result in a closed form for $C_{3,0}(-2, A)$ as displayed - and refined - in our Section 11.2. So now we know that $B_{6}(-2,-4)$ are both hyperclosed.

For the other direction away from $B_{6}(-4)$, we observe from (1-9) with $s \rightarrow-6$ that

$$
B_{6}(-8)=-\frac{\pi^{3}}{768}-\frac{5}{2} C_{4,0}(-6,2) .
$$

It turns out that we do not need to labor over the development of the $C$ evaluation here, because it is elementary from the very definition (1-3) that

$$
C_{m, 0}(s, A)=\frac{2}{s+2} \frac{\partial}{\partial A} C_{m, 0}(s+2, A),
$$

so that

$$
B_{6}(-8)=-\frac{\pi^{3}}{768}+\left.\frac{5}{4} \frac{\partial}{\partial A} C_{4,0}(-4, A)\right|_{A=2} ;
$$

thus $B_{6}(-8)$ is hyperclosed. Indeed, $C_{4,0}(-4, A)$ is hyperclosed by (4-1), while the derivative of a hyperclosed expression with respect to one algebraic parameter is itself hyperclosed, via the definitions of generalized hypergeometric functions and of the ring of hyperclosure.

Further, $B_{6}(-10,-12,-14, \ldots)$ can now be resolved recursively using (7-3) in tandem with (7-4) with $m=4$. This all leads to the following result.

Theorem 7.1. Every $B_{6}$ (even) is hyperclosed.

\section{$7.2 \quad B_{6}($ odd $)$}

In striking contrast to Theorem 7.1, we do not know a single $B_{6}$ (odd) in closed form. The best we can do at the current juncture is to obtain forms with yet new kinds of dangling integrals, e.g.,

$$
\begin{aligned}
B_{6}(-7)= & -\frac{\pi^{2}}{\sqrt{8}}+\sqrt{8} \pi \arctan \left(\frac{1}{\sqrt{2}}\right) \\
& -8 \int_{0}^{\pi / 4} \int_{0}^{\pi / 4} \frac{d t d u}{\sqrt{2+\sec ^{2} t+\sec ^{2} u}} .
\end{aligned}
$$

For a general parameter $A$ one has

$$
\begin{aligned}
& \int_{0}^{\pi / 4} \int_{0}^{\pi / 4} \frac{d t d u}{\sqrt{A+\sec ^{2} t+\sec ^{2} u}} \\
& \quad=\int_{0}^{1} \frac{\arctan \left(\sqrt{A+1+y^{2}} / \sqrt{A+3+y^{2}}\right)}{\left(1+y^{2}\right) \sqrt{A+1+y^{2}}} d y .
\end{aligned}
$$

Interestingly, we do know that this dangling integral for $A=1$ is equal to $\pi^{2} / 30$, on the basis of $(3-2)$; yet we 
do not yet know the $A=2$ evaluation that would yield $B_{6}(-7)$. It would surprise us somewhat if resolutions of such elusive box integrals involve more than trilogarithmic terms, based on heuristics we shall explore in Section 8.

Somewhat more can be said about $B_{6}$ (odd). By combining recurrences (1-8), (1-9), one can deduce

$$
\begin{aligned}
& 360 C_{2,0}(s, 4) \\
&=- 6 s^{2}(s-2)(s-4) B_{6}(s-6) \\
&+(s-2) s(s+2)(11 s+18) B_{6}(s-4) \\
&-(3+s)(4+s) \\
& \times\left\{2 s(3 s+8) B_{6}(s-2)-(s+5)(s+6) B_{6}(s)\right\} .
\end{aligned}
$$

We next observe that $C_{2,0}(s, A)$ is hyperclosed for odd integers $s$. We write

$$
C_{2,0}(s, A)=\frac{2}{s+2} M(s+2, A)-\frac{\pi}{2} \frac{A^{s / 2+1}}{s+2},
$$

where $M(s, A):=\int_{0}^{\pi / 4}\left(A+1+\tan ^{2} \theta\right)^{s / 2} d \theta$. We have the recurrence

$$
\begin{aligned}
& M(s+2, A)-A M(s, A) \\
& \quad=(A+1)^{s / 2}{ }_{2} F_{1}\left(\frac{1}{2},-\frac{s}{2} ; \frac{3}{2} ;-\frac{1}{A+1}\right),
\end{aligned}
$$

so that the hyperclosure of all $C_{2,0}($ odd, 4$)$ is ignited by a single evaluation, say

$C_{2,0}(-1,4)=4 \arctan (\sqrt{2} / 3)-\pi+\log 5-2 \log (\sqrt{6}-1)$.

One might guess that because the $C_{2,0}(s, 4)$ on the left of recurrence (7-5) is thus hyperclosed, the $B_{6}$ (odd) should be determined by $B_{6}$ at any three consecutive arguments. But this is not so: sometimes the coefficients in (7-5) vanish. By looking closely at cases such as $s=-1,-3,-5, \ldots$, one can establish the following:

Theorem 7.2. If $B_{6}(-7)$ is hyperclosed, then so are all of $B_{6}(-9,-11,-13, \ldots)$. If in addition $B_{6}(-3,-5)$ are hyperclosed, then so are all $B_{6}(\mathrm{odd})$.

Therefore, resolution of all $B_{6}$ (integer) would follow from hyperclosure of the three entities $B_{6}(-3,-5,-7)$; we remind ourselves that we do not know a closed form for any one of these three.

\section{THEORY OF HYPERDEGREE}

Based on previous box-integral research, together with the present treatment, an interesting pattern emerges in

\begin{tabular}{|c|c|c|}
\hline$n$ & $H\left(B_{n}(\right.$ even $\left.)\right) \leq$ & $H\left(B_{n}(\right.$ odd $\left.)\right) \leq$ \\
\hline 2 & 1 & 1 \\
3 & 2 & 1 \\
4 & 2 & 2 \\
5 & 3 & 2 \\
6 & 3 & $3 ?$ \\
7 & $4 ?$ & $3 ?$ \\
\hline & $\lceil n / 2\rceil ?$ & $\lfloor n / 2\rfloor ?$ \\
\hline
\end{tabular}

TABLE 1. Known bounds on hyperdegrees for box integrals. All hyperdegree entries without "?" are rigorous upper bounds (since we know the relevant closed forms). The immediate conjectures are that $H\left(B_{6}(\right.$ odd $\left.)\right) \leq 3$, that the hyperdegrees for $B_{7}$ are 4,3 , and that for general dimension $n>1$ we have the indicated floor and ceiling bounds.

regard to the "polylogarithmic degree" of various evaluations. We hereby define hyperdegree as a certain measure on the ring of hyperclosure, and mean this to be more general than polylogarithmic degree. We shall speak heuristically in what follows, because it is very hard to produce rigorous results in this area. One might say that in looking for hyperdegree patterns we are observing "evident hyperdegree" without proof. ${ }^{3}$

To define the hyperdegree $H(X)$ of a ring element $X$, we start with ring-generator evaluations $(2-1)$ and define $H(x)$ as the minimal degree of the denominator polynomial $q(n)$, over all hypergeometric expansions of $x$. For example (here $z$ denotes an algebraic number),

$$
H(z)=0, \quad H(\log z) \leq 1, \quad H\left(\operatorname{Li}_{n}(z)\right) \leq n .
$$

Note that we use $\leq$ sometimes because there can be exceptions, e.g., $\log 1=0$, and also because, again, we cannot always prove exact hyperdegrees. Some isolated cases can be proven, such as

$$
H(\pi)=1,
$$

whose proof is an instructive exercise. Generally speaking, when we obtain a closed form, we have an upper bound on the hyperdegree $H$.

Next we define the hyperdegree of a ring element $X$ using the symbolism

$$
X=\sum_{j}\left(\prod_{k} x_{j, k}\right)
$$

where the $x_{j, k}$ are an array-not necessarily rectangular - of generator evaluations, and both sum

\footnotetext{
${ }^{3}$ For example, is the hyperdegree of $\zeta(5)$ equal to 5 ? Not if $\zeta(5)$ is rational!
} 


$$
\begin{aligned}
G^{-}(b, c)= & \operatorname{Li}_{3}\left(\frac{b}{b-c}\right)-\operatorname{Li}_{3}\left(\frac{b+1}{b-c}\right)-\operatorname{Li}_{3}\left(\frac{b(c+1)}{b-c}\right)-\operatorname{Li}_{2}(-b) \log \left(\frac{b(c+1)}{b-c}\right) \\
& -\operatorname{Li}_{2}\left(\frac{b}{b-c}\right) \log \left(\frac{b(c+1)}{b-c}\right)+\operatorname{Li}_{2}\left(\frac{b+1}{b-c}\right) \log \left(\frac{(b+1)(c+1)}{b-c}\right)-\log b \operatorname{Li}_{2}\left(\frac{1}{c+1}\right) \\
& +\operatorname{Li}_{2}\left(\frac{1}{c+1}\right) \log \left(\frac{b(c+1)}{b-c}\right)+\operatorname{Li}_{2}\left(\frac{b(c+1)}{b-c}\right) \log \left(\frac{b(c+1)}{b-c}\right) \\
& -\frac{1}{6} \log ^{3}\left(-\frac{(b+1)(c+1)}{b-c}\right)-\frac{1}{2} \log ^{2}(b+1) \log \left(\frac{(b+1)(c+1)}{b-c}\right) \\
& -\frac{1}{2} \log (b+1) \log ^{2}\left(\frac{b(c+1)}{b-c}\right)+\frac{1}{2} \log (b+1) \log ^{2}\left(\frac{(b+1)(c+1)}{b-c}\right) \\
& -\frac{1}{2} \log c \log ^{2}\left(\frac{b(c+1)}{b-c}\right)+\frac{1}{2} \log \left(-\frac{(b+1) c}{b-c}\right) \log ^{2}\left(\frac{b(c+1)}{b-c}\right) \\
& +\frac{1}{2} \log (c+1) \log \left(\frac{b(c+1)}{b-c}\right)-\frac{1}{2} \log \left(-\frac{(b+1)(c+1)}{b-c}\right) \log ^{2}\left(\frac{(b+1)(c+1)}{b-c}\right) \\
& -\frac{1}{2} \log _{c} \log ^{2}\left(\frac{b}{b-c}\right)+\frac{1}{2} \log \left(\frac{b}{b-c}\right) \log \left(-\frac{c^{2}}{b-c}\right)+\frac{1}{2} \log ^{2}(c+1) \log ^{2}\left(\frac{b}{b-c}\right) \\
& +\frac{1}{2} \log { }^{2}\left(-\frac{(b+1)(c+1)}{b-c}\right) \log \left(\frac{(b+1)(c+1)}{b-c}\right)-\frac{1}{2} \log ^{2}\left(\frac{b+1}{b-c}\right) \log _{\left(\frac{c+1}{c-b}\right)} \\
& -\frac{1}{6} \pi^{2} \log ^{2}\left(-\frac{c+1}{b-c}\right)-\log \left(\frac{b}{b-c}\right) \log \left(-\frac{c}{b-c}\right) \log _{\left(\frac{b(c+1)}{b-c}\right)} \\
& +\log \left(\frac{c}{c+1}\right) \log \left(\frac{b}{b-c}\right) \log \left(\frac{b(c+1)}{b-c}\right) \\
& +\log \left(\frac{b+1}{b-c}\right) \log \left(\frac{(b+1)(c+1)}{b-c}\right) \log \left(\frac{c+1}{c-b}\right)+\operatorname{Li}_{3}(-b)+\frac{1}{6} \log ^{3}(b+1) \\
& +\operatorname{Li}_{3}\left(\frac{1}{c+1}\right) \cdot
\end{aligned}
$$

FIGURE 2. Explicit closed form for $G^{-}$.

and product here are finite. We define

$$
H(X):=\min _{()}\left(\max _{j}\left(\sum_{k} H\left(x_{j, k}\right)\right)\right),
$$

where $\min _{()}$means to take the minimum of all ring representations $x_{j, k}$ for $X$. (Such is necessary to guard against resonances such as $H\left(\pi^{2}-\pi^{2}\right)=0$, yet each component $\pi^{2}$ has hyperdegree 2.) The point of the rather recondite notation can be intuitively expressed thus: The hyperdegree of a ring element is the largest hyperdegree of an isolated product string, which is in turn (at most) the sum of the hyperdegrees of the $x_{j, k}$ members of said string. With such notions - admittedly not entirely rigorous - of hyperdegree, we have examples such as

$$
H\left(1+\log 7-\log 2 \log ^{2} 3-\pi^{3}+\operatorname{Li}_{3}(\sqrt{5}-2)\right) \leq 3,
$$

and this $H$ probably is in fact 3 . A specific and relevant box-integral example is

$$
\begin{aligned}
H & \left(B_{3}(-2)\right) \\
& =H\left(-3 G+\frac{3}{2} \pi \log (1+\sqrt{2})+3 \operatorname{Ti}_{2}(3-2 \sqrt{2})\right) \\
& \leq 2 .
\end{aligned}
$$

The implications of hyperdegree theory for box integrals can be gleaned from Table 1, which uses results from [Bailey et al. 09] together with our new results on $B_{5}, B_{6}$. Note that the development of Section 5.1 also adds substance to this conjecture.

\section{CONCLUSION}

Salient open issues include the following:

- Proof that some or all $B_{6}$ (odd) are hyperclosed.

- Evaluation of some $B_{n}(s)$ for $n \geq 7$, ideally of an infinite family such as $B_{n}(-n-1)$ or $B_{n+1}(-n)$ for $n \geq 6$.

- A better understanding of the structure of such evaluations.

Each of these is relevant also to other physically motivated classes of integrals [Bailey et al. 06].

Finally, one of the largest challenges for such computer-assisted analysis is to automate the process described in Appendices I and II so that results like (11-8) can be obtained by the computer with at most limited 


$$
\begin{aligned}
& G^{+}(b, c)=\frac{1}{2} \log \left(-\frac{c}{b-c}\right) \log ^{2}\left(\frac{b}{b-c}\right)-\frac{1}{2} \log \left(\frac{c}{c-1}\right) \log ^{2}\left(\frac{b}{b-c}\right)-\log \left(-\frac{b(c-1)}{b-c}\right) \log \left(-\frac{c}{b-c}\right) \log \left(\frac{b}{b-c}\right) \\
& +\log \left(-\frac{b(c-1)}{b-c}\right) \log \left(\frac{c}{c-1}\right) \log \left(\frac{b}{b-c}\right)-\frac{1}{2} \log (1-b) \log ^{2}\left(-\frac{b(c-1)}{b-c}\right) \\
& +\frac{1}{2} \log ^{2} 2 \log (1-b)+\frac{1}{2} \log (1-b) \log \left(-\frac{(b+1)(c-1)}{b-c}\right) \log \left(-\frac{(b+1)(c-1)}{4(b-c)}\right) \\
& +\frac{1}{2} \log ^{2}\left(-\frac{b(c-1)}{b-c}\right) \log \left(\frac{(b-1) c}{b-c}\right)-\frac{1}{2} \log ^{2}\left(-\frac{b(c-1)}{b-c}\right) \log \left(\frac{c}{c-1}\right) \\
& -\frac{1}{2} \log \left(-\frac{(b+1)(c-1)}{b-c}\right) \log \left(-\frac{(b+1)(c-1)}{4(b-c)}\right) \log \left(\frac{(b-1)(c+1)}{b-c}\right) \\
& -\frac{1}{2} \log ^{2} 2 \log \left(\frac{(b-1)(c+1)}{b-c}\right)+\frac{1}{2} \log ^{2}\left(\frac{b+1}{b-c}\right) \log \left(\frac{c+1}{c-1}\right) \\
& +\frac{1}{2} \log ^{2}\left(-\frac{(b+1)(c-1)}{b-c}\right) \log \left(\frac{c+1}{c-1}\right)+\log 2 \log \left(1+\frac{1}{b}\right) \log \left(\frac{c+1}{c-1}\right) \\
& +\log 2 \log b \log \left(\frac{c+1}{c-1}\right)-\log \left(\frac{b+1}{b-c}\right) \log \left(-\frac{(b+1)(c-1)}{b-c}\right) \log \left(\frac{c+1}{c-1}\right) \\
& -\log 2 \log \left(-\frac{(b+1)(c-1)}{b-c}\right) \log \left(\frac{c+1}{c-1}\right)+\frac{1}{2} \log ^{2} 2 \log \left(\frac{c+1}{c-1}\right) \\
& -\frac{1}{2} \log ^{2}\left(\frac{b+1}{b-c}\right) \log \left(\frac{c+1}{c-b}\right)+\log \left(\frac{b+1}{b-c}\right) \log \left(-\frac{(b+1)(c-1)}{b-c}\right) \log \left(\frac{c+1}{c-b}\right) \\
& -\log \left(-\frac{b(c-1)}{b-c}\right) \operatorname{Li}_{2}(b)+\log \left(-\frac{(b+1)(c-1)}{2(b-c)}\right) \operatorname{Li}_{2}\left(\frac{b+1}{2}\right)-\log b \operatorname{Li}_{2}\left(\frac{1}{1-c}\right) \\
& +\log \left(-\frac{b(c-1)}{b-c}\right) \operatorname{Li}_{2}\left(\frac{1}{1-c}\right)-\log \left(-\frac{b(c-1)}{b-c}\right) \operatorname{Li}_{2}\left(\frac{b}{b-c}\right) \\
& +\log \left(-\frac{(b+1)(c-1)}{b-c}\right) \operatorname{Li}_{2}\left(\frac{b+1}{b-c}\right)+\log \left(2+\frac{2}{b}\right) \operatorname{Li}_{2}\left(-\frac{2}{c-1}\right)+\log b \operatorname{Li}_{2}\left(-\frac{2}{c-1}\right)-\log \left(-\frac{(b+1)(c-1)}{b-c}\right) \operatorname{Li}_{2}\left(-\frac{2}{c-1}\right) \\
& -\log \left(-\frac{(b+1)(c-1)}{b-c}\right) \operatorname{Li}_{2}\left(-\frac{(b+1)(c-1)}{2(b-c)}\right)+\log 2 \operatorname{Li}_{2}\left(-\frac{(b+1)(c-1)}{2(b-c)}\right) \\
& +\log \left(-\frac{b(c-1)}{b-c}\right) \mathrm{Li}_{2}\left(\frac{b-b c}{b-c}\right)+\mathrm{Li}_{3}(b)-\mathrm{Li}_{3}\left(\frac{b+1}{2}\right)+\mathrm{Li}_{3}\left(\frac{1}{1-c}\right)+\mathrm{Li}_{3}\left(\frac{b}{b-c}\right)-\mathrm{Li}_{3}\left(\frac{b+1}{b-c}\right)-\mathrm{Li}_{3}\left(-\frac{2}{c-1}\right) \\
& +\operatorname{Li}_{3}\left(-\frac{(b+1)(c-1)}{2(b-c)}\right)-\mathrm{Li}_{3}\left(\frac{b-b c}{b-c}\right) \text {. }
\end{aligned}
$$

FIGURE 3. Explicit closed form for $G^{+}$.

human agency. The appendices provide invaluable test data for such projects - and one such project is currently being undertaken.

\section{APPENDIX I: THE FUNCTIONS $F^{ \pm}$AND $G^{ \pm}$}

The $F^{ \pm}$integrals resolve as

$$
\begin{aligned}
F^{+}(c)= & -\operatorname{Li}_{2}\left(\frac{1}{1-c}\right)+\operatorname{Li}_{2}\left(-\frac{2}{c-1}\right) \\
& +\log 2 \log \left(\frac{c+1}{c-1}\right), \\
F^{-}(c)= & -\operatorname{Li}_{2}\left(\frac{1}{c+1}\right) .
\end{aligned}
$$

It is already of interest that these two \pm forms seem to differ in complexity. In fact, trying an integrand factor $\log (1+s x)$ and expecting to take $s= \pm 1$ in a general integral involves a nontrivial limit for the $s=-1$ case. All of this difficulty can be traced to the well-known branch-cut peculiarity of the dilogarithm $\operatorname{Li}_{2}(z)$ on $z \in(+1,+\infty)$.
The $G^{ \pm}$integrals are far more intricate; and again, it is best to handle the \pm cases separately. The exact analytic forms displayed in Figures 2 and 3 can be gleaned from fundamental trilogarithmic formulas such as [Lewin 81, equation 8.111]. (It is again interesting that $G^{-}$is evidently less complex than $G^{+}$, although it is unclear what further symbolic reductions might be possible for either of $G^{ \pm}$.)

\section{APPENDIX II: EXPLICIT FORMS FOR $J(n)$, $0 \leq n \leq 4$, AND $C_{3,0}(-2, A)$}

The easiest value of $J$ to obtain is $J(0)$, which we may get directly from the original integral form. We write

$$
\begin{aligned}
J(0) & =2 \int_{0}^{\pi / 4} \int_{0}^{a} \frac{\log \left(a^{2}+b^{2}\right)}{\left(1+a^{2}\right)\left(1+b^{2}\right)} d b d a \\
& =-\int_{0}^{1} \frac{\log ^{2}\left(\frac{c^{2}+1}{2}\right) d c}{1-c^{2}}+\frac{\pi^{2}}{16} \log (2)+\frac{7}{4} \zeta(3)-\pi \mathrm{G} .
\end{aligned}
$$


Moreover,

$\int_{0}^{1} \frac{\log ^{2}\left(\frac{c^{2}+1}{2}\right) d c}{1-c^{2}}=\sum_{k=1}^{\infty} 2^{k} \frac{\sum_{j=1}^{k-1} 1 / j}{\left(\begin{array}{c}2 k \\ k\end{array}\right) k^{2}}=-\pi \mathrm{G}+\frac{21}{8} \zeta(3)$

so that

$$
J(0)=\frac{\pi^{2}}{16} \log 2-\frac{7}{8} \zeta(3) .
$$

We can evaluate $J(1)$ by much the same techniques as given above for $J(3)$. We obtain - in the limit - a tenthousand-character expression that with care reduces to the quantity displayed in Figure 4.

We refine this evaluation below. As we shall see, the expression of the integral $\mathcal{K}_{5}=J(3)$ is similar but significantly more complex, the original expression being roughly four times as long.

\subsection{Generalized Clausen Functions and Relatives}

To further resolve $J(1), J(2), J(3)$, and $J(4)$ one has to consider complex polylogarithms and generalized Clausen functions.

Let us define $\mathrm{Cl}_{2}(r, \theta):=\operatorname{ImLi}_{2}\left(r e^{i \theta}\right)$ as a counterpart to

$$
\begin{aligned}
\operatorname{Li}_{2}(r, \theta) & :=\operatorname{Re} L i_{2}\left(r e^{i \theta}\right) \\
& =-\frac{1}{2} \int_{0}^{r} \frac{\log \left(1-2 r \cos (\theta)+r^{2}\right)}{r} d \theta
\end{aligned}
$$

so that $\mathrm{Cl}_{2}(1, \theta)=\mathrm{Cl}_{2}(\theta)=\sum_{n \geq 1} \frac{\sin (n \theta)}{n^{2}}$. (We recall that $\mathrm{Cl}_{2}$ is a nonelementary Fourier series as opposed to $\mathrm{Li}_{2}(1, \theta)$.) Then, see [Lewin 81, A2.5.(1)], one obtains

$$
\begin{aligned}
\mathrm{Cl}_{2}(r, \theta)= & \frac{1}{2} \mathrm{Cl}_{2}(2 \omega)+\frac{1}{2} \mathrm{Cl}_{2}(2 \theta)-\frac{1}{2} \mathrm{Cl}_{2}(2 \omega+2 \theta) \\
& +w \log (r)
\end{aligned}
$$

where $\tan (\omega)=r \sin (\theta) /(1-r \cos (\theta))$.

Also for $r>0$ and integer $n, \operatorname{Li}_{2}(r, \theta)=\operatorname{Li}_{2}(r, 2 n \pi \pm$ $\theta)$ and $\mathrm{Cl}_{2}(r, \theta)=-\mathrm{Cl}_{2}(r,-\theta)$. Very usefully, for $0 \leq \theta<2 \pi$,

$$
\mathrm{Li}_{2}(r, \theta)+\mathrm{Li}_{2}(1 / r, \theta)=\frac{1}{2}(\pi-\theta)^{2}-\frac{1}{2} \log ^{2}(r)-\frac{\pi^{2}}{6} .
$$

We record the following useful Fourier series reductions:

$$
\begin{aligned}
\operatorname{Li}_{2} & \left(\tan \frac{\theta}{2}, \theta\right) \\
= & \frac{\theta^{2}}{4}+\frac{1}{2} \operatorname{Li}_{2}\left(\tan ^{2}\left(\frac{\theta}{2}\right)\right)-\frac{1}{4} \operatorname{Li}_{2}\left(\tan ^{2}\left(\frac{\theta}{2}\right)\right),
\end{aligned}
$$

as well as

$$
\begin{aligned}
\mathrm{Li}_{2}(2 \cos \theta, \theta)= & \left(\frac{\pi}{2}-\theta\right)^{2} \\
\mathrm{Li}_{2}(\cos \theta, \theta)= & \frac{1}{4} \mathrm{Li}_{2}\left(\cos ^{2} \theta\right)+\frac{1}{2}\left(\frac{\pi}{2}-\theta\right)^{2} \\
\mathrm{Li}_{2}(\sec \theta, \theta)= & \frac{5}{24} \pi^{2}-\frac{1}{4} \mathrm{Li}_{2}\left(\cos ^{2} \theta\right)-\frac{1}{2} \log ^{2}(\cos \theta) \\
& -\frac{\pi}{2} \theta \\
\mathrm{Li}_{2}\left(\frac{\sec \theta}{2}, \theta\right)= & \frac{\pi^{2}}{12}-\frac{1}{2} \log ^{2}(2 \cos \theta)-\frac{\theta^{2}}{2}
\end{aligned}
$$

In particular, using the final formula we obtain

$\operatorname{Li}_{2}\left(\frac{\sqrt{6}}{4}, \arctan \frac{1}{\sqrt{2}}\right)=\frac{\pi^{2}}{12}-\frac{1}{8} \log ^{2} \frac{8}{3}-\frac{1}{2} \arctan ^{2} \frac{1}{\sqrt{2}}$

and

$$
\begin{aligned}
\operatorname{Li}_{2} & \left(\frac{\sqrt{3}}{2}, \frac{\pi}{2}-\arctan \frac{1}{\sqrt{2}}\right) \\
& =\frac{\pi^{2}}{12}-\frac{1}{8} \log ^{2} \frac{4}{3}-\frac{1}{2}\left(\frac{\pi}{2}-\arctan \frac{1}{\sqrt{2}}\right)^{2} .
\end{aligned}
$$

Likewise, see [Lewin 81, A2.6], we notate $\operatorname{Li}_{3}(r, \theta):=$ $\operatorname{Re}^{2} \mathrm{Li}_{3}\left(r e^{i \theta}\right)$, so that in keeping with [Lewin 81], $\mathrm{Cl}_{3}(\theta):=\mathrm{Li}_{3}(1, \theta)$. In particular, [Lewin 81, A2.5] gives various functional equations for $\operatorname{Li}_{2}(r, \theta)$, and [Lewin 81, A2.6] gives various functional equations for $\mathrm{Li}_{3}(r, \theta)$. If we use the generalized tangent $T_{3}(\rho)=$ $\sum_{n \geq 0}(-1)^{n} \frac{\rho^{2 n+1}}{(2 n+1)^{3}}$, then

$$
\operatorname{Im} \operatorname{Li}_{3}\left(r e^{i \theta}\right)=T_{3}(\rho)-T_{3}(\rho, \tan \theta),
$$

where $\rho=r \sin \theta /(1-r \cos \theta)$ defines $T_{3}(\rho, \tan \theta)$. Also for $r>0$ we have $\operatorname{Li}_{3}(r, \theta)=-\mathrm{Li}_{3}(r,-\theta)$. Again, for $0 \leq \theta<2 \pi$, we have

$$
\begin{aligned}
\mathrm{Li}_{3} & (r, \theta)-\mathrm{Li}_{3}(1 / r, \theta) \\
& =-\frac{1}{6} \log ^{3} r+\frac{3(\pi-\theta)^{2}-\pi^{2}}{6} \log r .
\end{aligned}
$$

For example,

$$
\begin{aligned}
\operatorname{Li}_{3}\left(\sqrt{2}, \frac{\pi}{4}\right) & =\operatorname{Li}_{3}\left(\frac{1}{\sqrt{2}}, \frac{\pi}{4}\right)+\frac{11 \pi^{2}}{192} \log 2-\frac{1}{48} \log ^{3} 2 \\
& =\frac{35}{64} \zeta(3)+\frac{\pi^{2}}{32} \log 2
\end{aligned}
$$

We shall also exploit a consequence of the functional equation for the trilogarithm due to Landen 


$$
\begin{aligned}
J(1)= & \frac{9}{8} \pi^{2} \log 2-\frac{3}{2} \mathrm{G} \pi-\frac{1}{2} \log ^{2} 2 \log 3+\log ^{3} 2-\frac{7}{16} \pi^{2} \log 3 \\
& +\log ^{2}(1+\sqrt{2}) \log 2-\log ^{2}(1+\sqrt{2}) \log 3-2 \pi \arctan \sqrt{2} \log 2+\pi \arctan \sqrt{2} \log 3 \\
+ & \frac{1}{2} \pi \operatorname{Im}\left\{\operatorname{Li}_{2}(2-i+(1-i) \sqrt{2})+\operatorname{Li}_{2}(2-i-(1-i) \sqrt{2})\right\} \\
+ & \operatorname{Re}\left[(i \pi-2 i \arctan \sqrt{2}+3 \log 2-\log 3) \operatorname{Li} 2\left(\frac{1}{2}-\frac{1}{4} i \sqrt{2}\right)\right. \\
& +(2 i \pi-4 i \arctan \sqrt{2}+2 \log 3-4 \log 2) \operatorname{Li}_{2}\left(\frac{1}{2}-\frac{1}{2} i \sqrt{2}\right) \\
& +\left(\frac{3}{4} i \pi-i \arctan \sqrt{2}+\frac{1}{2} \log 2-\frac{1}{2} \log 3-\log (1+\sqrt{2}) \operatorname{Li}_{2}\left(\frac{1}{2}-\frac{3}{2} i-i \sqrt{2}\right)\right) \\
& +\left(-\frac{1}{4} i \pi+i \arctan \sqrt{2}+\frac{1}{2} \log 2-\frac{1}{2} \log 3+\log (1+\sqrt{2})\right) \operatorname{Li}_{2}\left(\frac{1}{2}-\frac{3}{2} i+i \sqrt{2}\right) \\
& \left.+2 \operatorname{Li}_{3}\left(\frac{1}{2}-\frac{1}{4} i \sqrt{2}\right)-4 \operatorname{Li}_{3}\left(\frac{1}{2}-\frac{1}{2} i \sqrt{2}\right)+\operatorname{Li} 3\left(\frac{1}{2}-\frac{3}{2} i+i \sqrt{2}\right)+\operatorname{Li}_{3}\left(\frac{1}{2}-\frac{3}{2} i-i \sqrt{2}\right)-2 \operatorname{Li}_{3}(1-i)\right] .
\end{aligned}
$$

FIGURE 4. One of our various resolutions of $J$ integrals.

$$
\begin{aligned}
2 C_{3,0}(-2, A)= & \frac{3}{2} \log ^{2} 2+\log 2 \log (A-1)-\pi \sqrt{A} \arctan \frac{1}{\sqrt{A}} \\
& +\operatorname{Li}_{2}\left(-\frac{A-1}{2}\right)+\operatorname{Li}_{2}\left(-\frac{2}{A-1}\right)-\operatorname{Li}_{2}\left(-\frac{A-1}{4}\right)-\operatorname{Li}_{2}\left(-\frac{4}{A-1}\right) \\
& +6 \operatorname{Im}\left[\operatorname{Li}_{2}\left(\frac{1+i}{1+\sqrt{A+2}}\right)+\operatorname{Li}_{2}\left(\frac{-1+i}{-1+\sqrt{A+2}}\right)\right] \\
+ & 2 \sqrt{A} \operatorname{Re}\left[\operatorname{Li}_{2}\left(\frac{(1+\sqrt{A})(\sqrt{A}+i \sqrt{A+2})}{2(A+1)}\right)-\operatorname{Li}_{2}\left(-\frac{(-1+\sqrt{A})(\sqrt{A}+i \sqrt{A+2})}{2(A+1)}\right)\right. \\
& +\operatorname{Li}_{2}\left(\frac{(1-\sqrt{A})(1-i \sqrt{A} \sqrt{A+2})}{(A+1)^{2}}\right)-\operatorname{Li}_{2}\left(\frac{(1+\sqrt{A})(1+i \sqrt{A} \sqrt{A+2})}{(A+1)^{2}}\right) \\
& \left.+\operatorname{Li}_{2}\left(\frac{(1+\sqrt{A})(1+i \sqrt{A})}{A+1}\right)-\operatorname{Li}_{2}\left(\frac{(1-\sqrt{A})(1-i \sqrt{A})}{A+1}\right)\right] .
\end{aligned}
$$

FIGURE 5. An important integral $C$, with free parameter $A$.

$$
\begin{aligned}
& C_{3,0}(-2, A)= \frac{1}{2} \log ^{2} 2-\frac{\pi^{2}}{8} \sqrt{A}-\frac{\pi}{2} \kappa \sqrt{A}+6 G-3 \mathrm{Cl}_{2}\left(\frac{\pi}{2}+\kappa\right)-3 \mathrm{Cl}_{2}\left(\frac{\pi}{2}-\kappa\right)+3 \kappa \log \left(\frac{\sqrt{A+2}+1}{\sqrt{A+2}-1}\right) \\
&+\sqrt{A}\left\{\operatorname{Li}_{2}\left(\frac{\sqrt{2}(\sqrt{A}+1)}{(A+1)^{3 / 2}}, \omega\right)-\operatorname{Li}_{2}\left(\frac{\sqrt{2}(\sqrt{A}-1)}{(A+1)^{3 / 2}}, \omega\right)+\operatorname{Li}_{2}\left(\frac{\sqrt{A}-1}{A+1}, \pi-\eta\right)\right. \\
&\left.\quad-\operatorname{Li}_{2}\left(\frac{\sqrt{A}+1}{A+1}, \eta\right)+\operatorname{Li}_{2}\left(\frac{\sqrt{A}+1}{\sqrt{A+1}}, \pi-\nu\right)-\operatorname{Li}_{2}\left(\frac{\sqrt{A}-1}{\sqrt{A+1}}, \nu\right)\right\} .
\end{aligned}
$$

FIGURE 6. Recasting of the $C$ integral.

[Lewin 81, A2.6 (7)]:

$$
\begin{aligned}
\mathrm{Li}_{3}(x) & +\mathrm{Li}_{3}(1-x)+\mathrm{Li}_{3}\left(\frac{-x}{1-x}\right) \\
= & \zeta(3)+\zeta(2) \log (1-x)+\frac{1}{2} \log ^{2}(1-x) \log x \\
& +\frac{1}{6} \log ^{3}(1-x) .
\end{aligned}
$$

This implies that for $0 \leq \omega \leq 2 \pi$ one has

$$
\begin{aligned}
\operatorname{Re} \mathrm{Li}_{3}\left(\frac{1}{2}+i \omega\right) \\
=\frac{1}{2} \zeta(3)-\frac{1}{2} \mathrm{Cl}_{3}(\sigma)+-\frac{1}{48} \log ^{3}\left(\frac{1+4 \omega^{2}}{4}\right) \\
\quad+\frac{1}{4}\left(\frac{\pi^{2}}{6}-\arctan ^{2}(2 \omega)\right) \log \left(\frac{1+4 \omega^{2}}{4}\right) .
\end{aligned}
$$




$$
\begin{aligned}
J(1)= & 2 \theta \pi \log \frac{4}{3}+\frac{\pi^{2}}{16} \log 3-\frac{7 \pi^{2}}{8} \log 2-\frac{1}{2} \log ^{2} 2 \log 3+\log ^{3} 2-\frac{3}{2} \mathrm{G} \pi+\log ^{2}(1+\sqrt{2}) \log \frac{2}{3} \\
& +2 \operatorname{Li}_{3}\left(\sqrt{2}, \frac{\pi}{4}\right)+\log \frac{8}{3} \operatorname{Li}_{2}\left(\frac{\sqrt{6}}{4}, \theta\right)-2 \log \frac{4}{3} \operatorname{Li}_{2}\left(\frac{\sqrt{3}}{2}, \frac{\pi}{2}-\theta\right)+2 \theta \mathrm{Cl}_{2}\left(\frac{\sqrt{6}}{4}, \theta\right)+4 \theta \mathrm{Cl}_{2}\left(\frac{\sqrt{3}}{2}, \frac{\pi}{2}-\theta\right) \\
& +\frac{\pi}{2} \mathrm{Cl}_{2}(\sqrt{6}+\sqrt{3}, \theta)+\frac{\pi}{2} \mathrm{Cl}_{2}(\sqrt{6}-\sqrt{3}, \theta)+\left(\frac{\pi}{4}+\theta\right) \mathrm{Cl}_{2}\left(\sqrt{3}+\frac{\sqrt{3}}{\sqrt{2}}, \frac{\pi}{4}+\theta\right)+\left(\frac{3 \pi}{4}+\theta\right) \mathrm{Cl}_{2}\left(\sqrt{3}-\frac{\sqrt{3}}{\sqrt{2}}, \frac{\pi}{4}-\theta\right) \\
& +4 \operatorname{Li}_{3}\left(\frac{\sqrt{3}}{2}, \frac{\pi}{2}-\theta\right)-2 \operatorname{Li}_{3}\left(\frac{\sqrt{6}}{4}, \frac{\pi}{2}-\theta\right)+\operatorname{Li}_{3}\left(\sqrt{3}+\frac{\sqrt{3}}{\sqrt{2}}, \frac{\pi}{4}-\theta\right)+\operatorname{Li}_{3}\left(\sqrt{3}-\frac{\sqrt{3}}{\sqrt{2}}, \frac{\pi}{4}-\theta\right) \\
& +\left(\frac{1}{2} \log \frac{2}{3}-\log (1+\sqrt{2})\right) \operatorname{Li}_{2}\left(\sqrt{3}+\frac{\sqrt{3}}{\sqrt{2}}, \frac{\pi}{4}+\theta\right)+\left(\frac{1}{2} \log \frac{2}{3}+\log (1+\sqrt{2}) \operatorname{Li}_{2}\left(\sqrt{3}-\frac{\sqrt{3}}{\sqrt{2}}, \frac{\pi}{4}-\theta\right)\right) .
\end{aligned}
$$

FIGURE 7. Recasting of $J(1)$.

$$
\begin{aligned}
J(1)= & \frac{\lambda^{2}}{2} \log \frac{3}{2}+\frac{\pi}{2}\left(\frac{\pi}{2}-\theta\right) \lambda+\pi \theta \log \frac{4}{3}-\frac{35}{32} \zeta(3)-\frac{1}{2} \log ^{3} 2+\frac{1}{4} \log ^{2} 2 \log 3+\frac{9 \pi^{2}}{32} \log \frac{3}{2} \\
& -\frac{\pi^{2}}{4} \log 2+2 \operatorname{Li}_{3}\left(\frac{\sqrt{6}}{4}, \theta-\frac{\pi}{4}\right)-4 \operatorname{Li}_{3}\left(\frac{\sqrt{3}}{2}, \theta\right)+\operatorname{Li}_{3}\left(\sqrt{3}-\sqrt{\frac{3}{2}}, \theta-\frac{\pi}{4}\right)+\operatorname{Li}_{3}\left(\sqrt{3}+\sqrt{\frac{3}{2}}, \theta-\frac{3 \pi}{4}\right) \\
& +\frac{\theta}{2} \mathrm{Cl}_{2}(4 \theta-\pi)+(2 \theta-\pi) \mathrm{Cl}_{2}(4 \theta)-3(2 \theta-\pi) \mathrm{Cl}_{2}(2 \theta)-\frac{\pi}{2} \mathrm{Cl}_{2}\left(2 \theta-\frac{\pi}{2}\right)+\frac{\pi}{2} \mathrm{Cl}_{2}\left(2 \theta+\frac{\pi}{2}\right)-G \pi
\end{aligned}
$$

FIGURE 8. Even more reduction of $J(1)$ is feasible.

Here $\sigma:=\arctan \left(\frac{4 \omega}{4 \omega^{2}-1}\right)$ is the principal value in $(-\pi, \pi]$.

The corresponding formula for the dilogarithm is

$$
\begin{aligned}
\operatorname{ReLi}_{2}\left(\frac{1}{2}+i \omega\right)= & \frac{\pi^{2}}{12}-\frac{1}{8} \log ^{2}\left(\frac{1+4 \omega^{2}}{4}\right) \\
& -\frac{1}{2} \arctan ^{2}(2 \omega)
\end{aligned}
$$

\subsection{A Closed Form for $C_{3,0}(-2, A)$}

We now have the requisite tools to produce an explicit closed form for $C_{3,0}(-2, A)$ as required to establish hyperclosure of all $B_{6}$ (even). We recall that

$$
C_{3,0}(-2, A)=\int_{[0,1]^{3}} \frac{d x d y d z}{A+x^{2}+y^{2}+z^{2}} .
$$

The formula that comes out of our intricate integration, using the techniques behind Lemma 4.1, is displayed in Figure 5 .

We next rewrite the dilogarithmic terms of $\mathrm{Li}_{2}(r, \theta)$ and $\mathrm{Cl}_{2}(r, \theta)$ values. For (a) in the formula we appeal twice to $(11-4)$ with $\theta=\pi$. We discover that

$$
\begin{array}{r}
\mathrm{Li}_{2}\left(-\frac{A-1}{2}\right)+\mathrm{Li}_{2}\left(-\frac{2}{A-1}\right)-\mathrm{Li}_{2}\left(-\frac{A-1}{4}\right) \\
-\mathrm{Li}_{2}\left(-\frac{4}{A-1}\right)=\frac{3}{2} \log ^{2} 2-\log 2 \log (A-1) .
\end{array}
$$

We write (b) as

$$
\begin{aligned}
6 \operatorname{Im}_{2} & \left(\frac{1+i}{1+\sqrt{A+2}}\right)+6 \operatorname{Im~Li}_{2}\left(\frac{-1+i}{-1+\sqrt{A+2}}\right) \\
= & 6 \mathrm{Cl}_{2}\left(\frac{\sqrt{2}}{1+\sqrt{A+2}}, \frac{\pi}{4}\right) \\
& -6 \mathrm{Cl}_{2}\left(\frac{\sqrt{2}}{-1+\sqrt{A+2}}, \frac{3 \pi}{4}\right) \\
= & 6 G-3 \mathrm{Cl}_{2}\left(\frac{\pi}{2}+\kappa\right)-3 \mathrm{Cl}_{2}\left(\frac{\pi}{2}-\kappa\right) \\
& +3 \kappa \log \left(\frac{\sqrt{A+2}+1}{\sqrt{A+2}-1}\right)
\end{aligned}
$$

where $\kappa:=2 \arctan \left(\frac{1}{\sqrt{A+2}}\right)$.

Similarly, (c) is actually three pairs of $\mathrm{Li}_{2}(r, \theta)$ values with equal angles and twisted moduli. Let $\nu:=\arctan (\sqrt{A}), \eta=: \arctan (\sqrt{A(A+2)}), \omega:=$ $\arctan (\sqrt{(A+2) / A})$. Note that $2 \omega+\eta=\pi$.

Then putting everything together for $A \geq 1$, we have the formula displayed in Figure 6.

This form is especially attractive when $A=1$, so that $\kappa=\omega=\eta=\pi / 3$ and $\nu=\pi / 4$. On noting that

$$
4 G=3 \mathrm{Cl}_{2}\left(\frac{\pi}{6}\right)+3 \mathrm{Cl}_{2}\left(\frac{5 \pi}{6}\right) \mathrm{Li}_{2}\left(\sqrt{2}, \frac{\pi}{4}\right)=\frac{\pi^{2}}{16}
$$




$$
\begin{aligned}
J(2)= & \frac{1}{108} \Psi^{\prime}\left(\frac{1}{3}\right) \sqrt{3} \pi+\frac{1}{108} \Psi^{\prime}\left(\frac{1}{6}\right) \pi \sqrt{3}-\frac{2}{81} \pi^{3} \sqrt{3}+\left(\frac{5}{12} \log (1+\sqrt{3})+\frac{1}{24} \log 2\right) \pi^{2} \\
& -\frac{53}{48} \zeta(3)-\mathrm{G} \pi+\operatorname{Re} \operatorname{Li}_{3}\left(\frac{1-i+\sqrt{3}+i \sqrt{3}}{2(\sqrt{3}+1)}\right)+\operatorname{Re} \operatorname{Li} 3\left(\frac{1+i-\sqrt{3}+i \sqrt{3}}{2(\sqrt{3}-1)}\right)-\frac{\pi}{2} \operatorname{Im} \operatorname{Li}_{2}\left(\frac{i+\sqrt{3}}{\sqrt{3}-1}\right) \\
& +\frac{5 \pi}{12} \operatorname{Im~Li} 2\left(\frac{-1+i+\sqrt{3}+i \sqrt{3}}{2(\sqrt{3}-1)}\right)-\frac{\pi}{12} \operatorname{ImLi}_{2}\left(\frac{1+i+\sqrt{3}-i \sqrt{3}}{2(\sqrt{3}+1)}\right)+\frac{\pi}{2} \operatorname{Im} \operatorname{Li}_{2}\left(\frac{i+\sqrt{3}}{\sqrt{3}+1}\right) .
\end{aligned}
$$

FIGURE 9. Closed form for integral $J(2)$.

$$
\begin{aligned}
J(2)= & \frac{\pi}{4} \sqrt{3} L_{-3}(2)+\frac{5}{12} \pi^{2} \log (1+\sqrt{3})+\frac{1}{24} \pi^{2} \log 2 \\
& -\frac{53}{48} \zeta(3)-G \pi+\operatorname{Li}_{3}\left(\frac{\sqrt{3}-1}{\sqrt{2}}, \frac{\pi}{12}\right) \\
& -\mathrm{Li}_{3}\left(\frac{\sqrt{3}+1}{\sqrt{2}}, \frac{5 \pi}{12}\right)+\frac{\pi}{2} \mathrm{Cl}_{2}\left(\frac{\sqrt{3}-1}{2}, \frac{\pi}{6}\right)-\frac{\pi}{2} \mathrm{Cl}_{2}\left(\frac{\sqrt{3}+1}{2}, \frac{\pi}{6}\right)+\frac{5 \pi}{12} \mathrm{Cl}_{2}\left(\frac{\sqrt{3}+1}{\sqrt{2}}, \frac{5 \pi}{12}\right)-\frac{\pi}{12} \mathrm{Cl}_{2}\left(\frac{\sqrt{3}-1}{\sqrt{2}}, \frac{\pi}{12}\right) .
\end{aligned}
$$

FIGURE 10. An alternative closed form for $J(2)$.

$$
\begin{aligned}
R_{5}= & \lambda\left(\operatorname{Li}_{2}\left(\frac{\sqrt{15}}{6}-\frac{\sqrt{30}}{12}, \theta+\tau\right)-\operatorname{Li}_{2}\left(\frac{\sqrt{15}}{6}+\frac{\sqrt{30}}{12}, \tau-\theta\right)-2 \operatorname{Li}_{2}\left(\frac{\sqrt{30}}{6}, \theta+\tau-\pi\right)\right) \\
& +2 \lambda\left(\operatorname{Li}_{2}\left(\frac{\sqrt{10}}{3}, \theta-\tau\right)-\operatorname{Li}_{2}\left(\frac{2}{\sqrt{3}}, \tau+\frac{3 \pi}{4}\right)+\operatorname{Li}_{2}\left(\frac{2}{\sqrt{3}}, \tau-\frac{\pi}{4}\right)+\operatorname{Li}_{2}\left(\frac{\sqrt{30}}{6}, \tau-\theta\right)-\operatorname{Li}_{2}\left(-\frac{\sqrt{2}-1}{2}\right)\right) \\
& +2 \lambda\left(\operatorname{Li}_{2}\left(\frac{\sqrt{10}}{6}+\frac{\sqrt{5}}{6}, \pi-\theta-\tau\right)-\operatorname{Li}_{2}\left(\frac{\sqrt{10}}{3}, \theta+2 \tau-\pi\right)-\operatorname{Li}_{2}\left(\frac{1}{\sqrt{3}}+\frac{1}{\sqrt{6}}, \tau-\frac{\pi}{4}\right)\right) \\
& +\frac{1}{2}\left(\operatorname{Li}_{2}\left(\frac{\sqrt{15}}{6}-\frac{\sqrt{30}}{12}, \theta+\tau\right)+\operatorname{Li}_{2}\left(\frac{\sqrt{15}}{6}+\frac{\sqrt{30}}{12}, \tau-\theta\right)\right) \log \left(\frac{24}{5}\right)-\operatorname{Li}_{3}\left(\frac{\sqrt{10}}{6}-\frac{\sqrt{5}}{6}, \theta-2 \tau\right) \\
& -\operatorname{Li}_{3}\left(\frac{\sqrt{10}}{6}+\frac{\sqrt{5}}{6}, \theta+2 \tau-\pi\right)-\operatorname{Li}_{3}\left(\frac{\sqrt{15}}{6}-\frac{\sqrt{30}}{12}, \theta+\tau\right)+\operatorname{Li}\left(\frac{\sqrt{15}}{6}+\frac{\sqrt{30}}{12}, \tau-\theta\right) \\
& +\operatorname{Li}_{3}\left(\frac{1}{\sqrt{3}}-\frac{1}{\sqrt{6}}, \tau-\frac{3 \pi}{4}\right)+\operatorname{Li}_{3}\left(\frac{1}{\sqrt{3}}+\frac{1}{\sqrt{6}}, \frac{\pi}{4}-\tau\right)+\operatorname{Li}_{3}\left(\frac{\sqrt{10}}{6}, \frac{\pi}{4}-\theta\right)+\operatorname{Li}_{3}\left(\frac{\sqrt{10}}{2}, \frac{3 \pi}{4}-\theta\right) \\
& -2 \operatorname{Li}_{3}\left(\frac{\sqrt{5}}{2}, \theta\right)+\operatorname{Li}_{3}\left(-\frac{\sqrt{2}-1}{2}\right)-\operatorname{Li}_{3}(2 \sqrt{2}-2) .
\end{aligned}
$$

FIGURE 11. A component of the elusive $\mathcal{K}_{5}$ integral.

$$
\begin{aligned}
L_{5}= & -\frac{7}{16} \zeta(3)-\frac{1}{2} G \pi+\frac{7}{6} \lambda^{3}+\left(\frac{1}{2} \log 5-\frac{1}{2} \log 3-2 \log 2\right) \lambda^{2}+\frac{3 \pi}{2}\left(\log 2-\frac{2}{3} \log 5+\log 3\right) \tau, \\
& +\left(\theta^{2}-3 \tau^{2}+3(4 \tau-\pi) \theta-\frac{23}{48} \pi^{2}+\frac{1}{4} \log ^{2} 2+\frac{3}{4} \log ^{2} 3-\log 3 \log 5+\frac{1}{4} \log ^{2} 5+\left(\frac{3}{2} \log 3-\log 5\right) \log 2\right) \lambda \\
& +\left(\frac{1}{2} \log 3-\frac{1}{2} \log 5+\frac{3}{2} \log 2\right) \theta^{2}-\frac{\pi}{2}\left(\frac{3}{2} \log 3+\log 2\right) \theta-\left(\frac{7}{2} \log 2-2 \log 5+\frac{7}{2} \log 3\right) \tau^{2} \\
& +3 \log ^{3} 2+\frac{5}{24} \log ^{3} 3-\frac{1}{8} \log ^{3} 5-\left(\frac{1}{2} \log 3 \log 5+3 \log ^{2} 2+\frac{3}{8} \log ^{2} 5\right) \log 3 \\
& +\left(\frac{11}{8} \log ^{2} 3-\frac{9}{4} \log 3 \log 5+\frac{9}{8} \log ^{2} 5-\frac{25}{8} \log 2 \log 5\right) \log 2+\left(\frac{23}{6} \log 5-\log 2\right) \frac{\pi^{2}}{16}
\end{aligned}
$$

FIGURE 12. Another component of $\mathcal{K}_{5}$. 


$$
\begin{aligned}
C_{5}= & \theta\left(\frac{1}{2} \mathrm{Cl}_{2}(\pi-4 \theta)+2 \mathrm{Cl}_{2}(2 \theta-\pi)-\mathrm{Cl}_{2}(4 \tau+2 \theta)+\mathrm{Cl}_{2}(4 \tau-2 \theta)-\mathrm{Cl}_{2}(2 \tau-2 \theta)+\mathrm{Cl}_{2}(2 \tau+2 \theta)\right) \\
+ & \frac{\pi}{4}\left(2 \mathrm{Cl}_{2}(2 \theta-\pi)-2 \mathrm{Cl}_{2}\left(\frac{\pi}{2}+2 \theta\right)+\mathrm{Cl}_{2}\left(\frac{\pi}{2}-2 \theta\right)+9 \mathrm{Cl}_{2}\left(2 \tau-\frac{\pi}{2}\right)-9 \mathrm{Cl}_{2}\left(2 \tau+\frac{\pi}{2}\right)\right. \\
& \left.\quad+\mathrm{Cl}_{2}(4 \tau-2 \theta)-\mathrm{Cl}_{2}(4 \tau+2 \theta)\right)
\end{aligned}
$$

FIGURE 13. Final component of $\mathcal{K}_{5}$.

and that

$$
\begin{aligned}
\mathrm{Li}_{2}\left(1, \frac{\pi}{4}\right) & =\frac{11 \pi^{2}}{192} \\
\mathrm{Li}_{2}\left(1, \frac{3 \pi}{4}\right) & =\frac{13 \pi^{2}}{192}
\end{aligned}
$$

it reduces to $2 C_{3,0}(-2,1)=B_{4}(-2)=\pi \log (2+\sqrt{3})-$ $2 G+\pi^{2} / 8$, as obtained in [Bailey et al. 09].

We can similarly rework (4-1) as follows:

$$
\begin{aligned}
C_{4,0}(-4, A)= & \frac{\pi^{2}}{16} \log A-\frac{\pi}{2} G+\frac{\pi}{4} \mathrm{Cl}_{2}\left(\frac{\sqrt{A+1}+1}{\sqrt{A}-1}, \frac{\pi}{2}\right) \\
& +\frac{\pi}{4} \mathrm{Cl}_{2}\left(\frac{\sqrt{A}-1}{\sqrt{A}+1}, \frac{\pi}{2}\right)-J(A+2) .
\end{aligned}
$$

This may again be further refined in terms of classical Clausen values.

\subsection{The Promised Closed Forms for $J$}

$\mathbf{J}(\mathbf{1})$. With this notation in hand, on setting $\theta:=$ $\arctan (1 / \sqrt{2})$, we may rewrite $J(1)$ as displayed in Figure 7.

Moreover, (11-3) allows for all $\mathrm{Cl}_{2}$ values to be expressed in terms of the classical Clausen function. We have placed the terms at the top for which we have thus shown reduction to elementary constants and classical polylogarithms or Clausen functions. Further simplification is possible of terms such as $\log \frac{8}{3} \operatorname{Li}_{2}\left(\frac{\sqrt{6}}{4}, \theta\right)$ and $2 \log \frac{4}{3} \operatorname{Li}_{2}\left(\frac{\sqrt{3}}{2}, \frac{\pi}{2}-\theta\right)$.

We now opt to set $\lambda:=\log (1+\sqrt{2})$ and $\theta:=$ $\arctan \sqrt{2}$. We eventually arrive at the expression of Figure 8 .

Each level of simplification reveals more structure. We now note that each $\mathrm{Li}_{3}$ value is of the form $\operatorname{Li}_{3}\left(\frac{\sec \tau}{2}, \tau\right)$, where $\tau:=\theta+\frac{k \pi}{4}, 0 \leq k \leq 3$. Each of these may be resolved by an application of $(11-5)$. This leads to

$$
\begin{aligned}
J(1)= & \frac{\pi}{2} \mathrm{Cl}_{2}\left(2 \theta-\frac{\pi}{2}\right)+\frac{\pi}{2} \mathrm{Cl}_{2}\left(2 \theta+\frac{\pi}{2}\right) \\
& -\frac{\theta}{2} \mathrm{Cl}_{2}(4 \theta-\pi)+(2 \theta-\pi) \mathrm{Cl}_{2}(4 \theta) \\
& -3(2 \theta-\pi) \mathrm{Cl}_{2}(2 \theta)+2 \mathrm{Cl}_{3}(2 \theta-\pi)-\mathrm{Cl}_{3}(2 \theta) \\
& -\frac{1}{2} \mathrm{Cl}_{3}\left(2 \theta+\frac{\pi}{2}\right)-\frac{1}{2} \mathrm{Cl}_{3}\left(2 \theta-\frac{\pi}{2}\right)-G \pi \\
& -\frac{35}{32} \zeta(3) .
\end{aligned}
$$

$\mathbf{J}(2)$. Surprisingly, $J(2)$ is significantly simpler, because of the specific angles engaged, though one has to approach the removable singularity carefully. This again leads to a very large symbolic expression, which reduces to the formula of Figure 9

Again, the dilog terms can be nicely resolved as Clausen values, and the trilogarithms can be manipulated as above. This produces the expression in Figure 10 , which, remarkably, reduces to a single line:

$J(2)$

$$
=\frac{\pi^{2}}{8} \log 2-\frac{7}{48} \zeta(3)+\frac{11}{24} \pi \mathrm{Cl}_{2}\left(\frac{\pi}{6}\right)-\frac{29}{24} \pi \mathrm{Cl}_{2}\left(\frac{5 \pi}{6}\right) .
$$

We can use (11-6) to replace $\mathrm{Cl}_{2}\left(\frac{5 \pi}{6}\right)$ by $\frac{4}{3} G-\mathrm{Cl}_{2}\left(\frac{\pi}{6}\right)$. We can also substitute

$$
\mathrm{Cl}_{2}\left(\frac{\pi}{6}\right)=\frac{G}{3}+\frac{3 \sqrt{3}}{16} L_{-3}(2),
$$

where $L_{-3}(2)$ is the primitive $L$-series modulo 3 . Thus, alternatively we have

$$
J(2)=\frac{5 \pi}{16} \sqrt{3} L_{-3}(2)-\frac{\pi}{2} G-\frac{7}{48} \zeta(3)+\frac{\pi^{2}}{8} \ln 2 .
$$

$\mathbf{J}(\mathbf{3})$. Using the decomposition in Theorem 5.1, an explicit polylogarithmic form of $\mathcal{K}_{5}=J(3)$ was obtained in 
Mathematica. Said expression was roughly 30,000 characters long - or 62,000 when converted to a Maple expression. Either of these language forms comes down to about 50 kilobits of "expression entropy," as discussed in Section 6. The challenge is to find a much smaller expression. Write

$$
J(3)=\mathcal{K}_{5}=R_{5}+C_{5}+L_{5},
$$

where $R_{5}$ comprises the real trilog and dilog terms, $C_{5}$ comprises the pure Clausen terms, and $L_{5}$ collects the rest. Then, after a very large amount of symbolic work exploiting many formulas in [Lewin 81], on setting $\lambda:=\log (1+\sqrt{2}), \theta:=\arctan 2$, and $\tau:=\arctan \sqrt{2}$, we obtain for $R_{5}$ the expression in Figure 11.

Likewise, the constant and pure logarithmic coefficients $L_{5}$ reduce to Figure 12 . We also have the expression for $C_{5}$ shown in Figure 13.

Formula (11-10) was numerically checked to $200 \mathrm{dec}-$ imal places. Given the final form for $J(1), J(2)$, it is suspected that $J(3)$ can be further simplified. Indeed, most of the $\mathrm{Li}_{3}$ terms are susceptible to (11-5), with consequent simplification of $L_{5}$.

$\mathbf{J}(4)$. For $J(4)$ the corresponding expression has been reduced from roughly 100 kilobits of expression entropy down to expressions similar to but somewhat longer than those for $J(3)$. We now use angles $\arctan \sqrt{5}$ and $\arctan \sqrt{15}$ and noninteger logarithms $\log (1+\sqrt{3})$ and $\log (1+\sqrt{15})$.

In each case the angles are coupled to those engaged in the expression of Figure 6 for $A-1$.

\section{ACKNOWLEDGMENTS}

We wish to thank D. Bailey and A. Kaiser for many useful discussions and for computational confirmation of some the results. The first author's research is supported by the Australian Research Council.

\section{REFERENCES}

[Anderson et al. 76] R. Anderssen, R. Brent, D. Daley, and P. Moran. "Concerning $\int_{0}^{1} \cdots \int_{0}^{1}\left(x_{1}^{2}+\cdots x_{n}^{2}\right)^{\frac{1}{2}} d x_{1} \cdots d x_{n}$ and a Taylor Series Method." SIAM J. Applied Math. 30 (1976), 22-30.

[Bailey et al. 06] D. H. Bailey, J. M. Borwein, and R. E. Crandall. "Integrals of the Ising Class." J. Phys. A. 39 (2006), 12271-12302. Available online (http://crd.lbl.gov/ $\sim$ dhbailey/dhbpapers/Ising.pdf).

[Bailey et al. 07] D. H. Bailey, J. M. Borwein, and R. E. Crandall. "Box integrals." Journal of Computational and Applied Mathematics 206 (2007), 196-208.

[Bailey et al. 09] D. H. Bailey, J. M. Borwein, and R. E. Crandall. "Advances in the Theory of Box Integrals." Mathematics of Computation 79 (2010), 1839-1866.

[Borwein and Crandall 10] J. M. Borwein and R. E. Crandall, "Closed Forms: What They Are and Why We Care." To appear in Notices of the Amer. Math. Soc., 2010.

[Crandall 10] R. E. Crandall. "Theory of Box Series." Available online (http://www.perfscipress.com/papers/FastBn_ psipress.pdf), 2010.

[Lewin 81] Leonard Lewin. Polylogarithms and Associated Functions. Amsterdam: North Holland, 1981.

[Sloane 09] N. J. A. Sloane, editor. The On-Line Encyclopedia of Integer Sequences. Available at www.research.att. com/ njas/sequences/, 2009.

Jonathan M. Borwein, Centre for Computer Assisted Research Mathematics and Its Applications (CARMA), University of Newcastle, Callaghan, New South Wales, 2308, Australia (jon.borwein@gmail.com)

O-Yeat Chan, 10221 Hollymount Drive, Richmond, BC, V7E 4T5, Canada (math@oyeat.com)

R. E. Crandall, Center for Advanced Computation, Reed College, Portland OR (crandall@reed.edu)

Received January 24, 2010; accepted March 15, 2010. 